\title{
Characterization and Analysis of Propagation Time Delay Range within NigComSat-1R Footprints
}

\author{
A. T. Ajiboye*, A. O. Yusuf, A. R. Ajayi \\ Department of Computer Engineering, University of Ilorin, Ilorin, Nigeria.
}

\begin{abstract}
NigComSat-1R could be part of Networked Control Systems (NCSs) to link plants, controllers, sensors and actuators which may be distributed within the satellite footprints. Associated with NCSs is location-dependent time delay which can drastically reduce system Quality of Performance (QoP), or in the worst case lead to system instability. To ameliorate these effects, the network delay should be taken into consideration at design stage. In order to achieve this, the ranges of propagation time delay incurred within a particular footprint or between two footprints of NigComSat-1R are modelled, simulated, characterized and analyzed. It was observed that the minimum and maximum possible time delays between the boundary of NigComSat-1R footprints and the satellite are $0.1193 \mathrm{sec}$ and $0.141 \mathrm{sec}$ respectively. Also, the minimum possible propagation time delay between any two footprints is that between C-band ECOWAS 1 beam and itself with value of $0.2386 \mathrm{sec}$ while the maximum possible propagation time delay between any two footprints is that between L-band Navigation payload L1 beam and itself or L-band Navigation payload L5 beam and itself with value of $0.2832 \mathrm{sec}$.
\end{abstract}

KEYWORDS: Footprint, networked control system, Nigcomsat-1R, propagation time delay, quality of performance.

\section{INTRODUCTION}

NigComSat-1R was the next satellite launched after disappearance of the first Nigeria satellite. NigComSat-1R was launched for the purpose of providing security, social, and industrial development within African sub-regions and Nigeria in particular. All the regions under NigComSat-1R footprint, which include the whole of Africa, some part of Asia and Europe are being served by this satellite in the area of Information and Communication Technology (ICT) (Nwajiobi, 2012).

NigComSat-1R is positioned at $42.5^{\circ}$ East in geostationary orbit and could be part of NCSs to link plants, controllers, sensors and actuators which may be distributed all over the regions within the footprints. Designing and implementation of control systems whereby the link among the control elements is via satellite have

become necessary in Nigeria because of the inherent advantages associated with NCSs.

The advantages of NCS over point-to-point system include reduced cost of wiring, ease of diagnosis and maintenance and increased system agility (Vatanski et al., 2006). Another advantage is its applicability in hazardous or security-challenged regions.

Using satellite as medium of communication among control system elements normally introduce time delay in the transmission of information between controller and plant, and between sensor and controller which may complicate system design and analysis (Vatanski et al., 2006). The propagation *Corresponding author: ajiboye.at@unilorin.edu.ng delays associated with NCSs can degrade control system performance and in worst cases, it may destabilize the system if not properly considered during system design (Kumar \& Kumar, 2013).Time delay is harmful to control system when the performance criterion is based on stability margin. However, it becomes advantageous when the performance criterion is based on tracking error particularly if the system under consideration is a type I system with ramp reference inputs and the time delays involved is consistent, predictable and within tolerable range (Khan, Tilbury, \& Moyne, 2008).

Whatever the effects of propagation delay on any given system are, one should be able to determine and characterize its range so that its effects on system QoP can be quantified and compensated for if need be.

Goyal et al., (1999) modelled the propagation delay between two points on the earth surface via constellation of many satellites as the sum of the delays between the sending node and the first satellite; inter-satellite propagation delays and the delay between the last satellites and destination node. Only one satellite, NigComSat-1R was involved in this research work, therefore, the inter-satellite link propagation delays does not exist.

The modelling, simulation, characterization and analysis of the following scenarios are presented in this article: (i) range of propagation delay between any point in a particular satellite footprint and the satellite, (ii) the range of propagation delay between any two points within a particular footprint via satellite and (iii) the range of propagation delay

doi: http://dx.doi.org/10.4314/njtd.v16i3.3 
between any two points in two different footprints via satellite. Both the minimum and maximum possible propagation time delays were determined. Based on this, prospective control system designer will know the range of propagation delays in any footprint or between any footprints of interest and consequently be able to come up with a stable system with good QoP.

\section{PROPAGATION DELAY MODEL FOR SATELLITE COMMUNICATION SYSTEMS}

This section describes the modelling of propagation delay incurred by sending data between two earth base stations either within the same footprint region or between two different footprint regions of the concerned geostationary satellite.

\section{A. Model of Propagation Delay between a Point on Earth Surface and Geostationary Satellite}

The distance between point $\mathrm{P}$ on earth surface and any geostationary satellite denoted by $d$ as shown in Figure 1 is given by (Ibiyemi \& Ajiboye, 2012a, 2012b; Kolawole, 2002) as

$$
d=\sqrt{D^{2}+R^{2}-2 D R \cos (\alpha) \cos (\Delta)}
$$

where:

$$
\Delta=\Delta_{E S}-\Delta_{S}
$$

$R=$ radius of the earth in $\mathrm{km}$;

$D=$ the sum of the radius of the earth and satellite altitude in $\mathrm{km}$;

$\alpha=$ site latitude in degrees;

$\Delta=$ difference between subsatellite point longitude and site longitude in degrees;

$\Delta_{E S}=$ the angle of longitude of the sending node on the earth surface in degrees;

$\Delta_{S}=$ the angle of longitude of the subsatellite point in degrees.

$\Delta_{\mathrm{S}}$ and $\Delta_{\mathrm{ES}}$ are assumed to be positive if they fall to the East of the Greenwich Meridian and negative if they fall to the West; while $\alpha$ is assumed to be positive for latitudes in the North of the equator and negative for the latitude in the South of the equator.

The model equation of the propagation delay, $T_{p}$ between any point within a footprint and the satellite, assuming a negligible transponder delay, is given by eqn (2).

$$
T_{p}=\frac{d}{c}
$$

$c$ is the speed of light in $\mathrm{km} / \mathrm{sec}$ for clear-sky weather conditions.

\section{B. Modelling of Propagation Delay between Two Points on Earth Surface via Geostationary Satellite}

The model equation for propagation delay between two points on earth surface via a geostationary satellite can be obtained by taking the following steps:

Determine the distance between each of these points and the geostationary satellite,

Divide the determined distance by signal speed to obtain the delay between each of these points and the satellite

Then add the determined delay between these two points and the satellite together

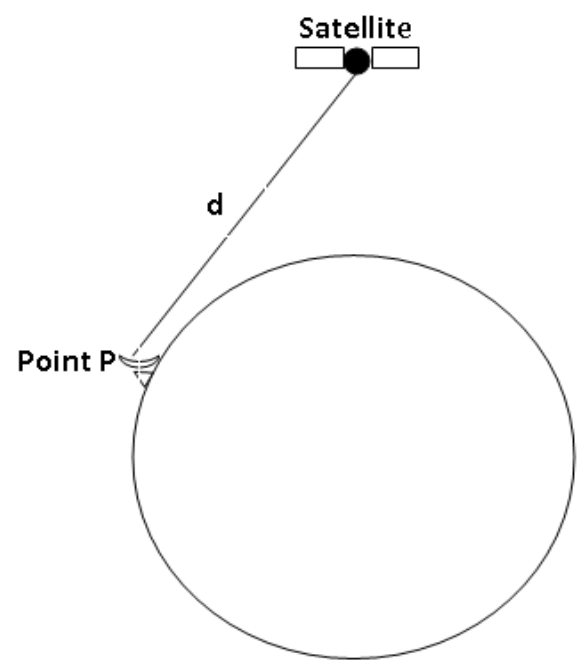

Figure 1: Distance between point $P$ on earth surface and geostationary satellite.

The distance between point 1 and point 2 on earth surface via geostationary satellite is shown in Figure 2:

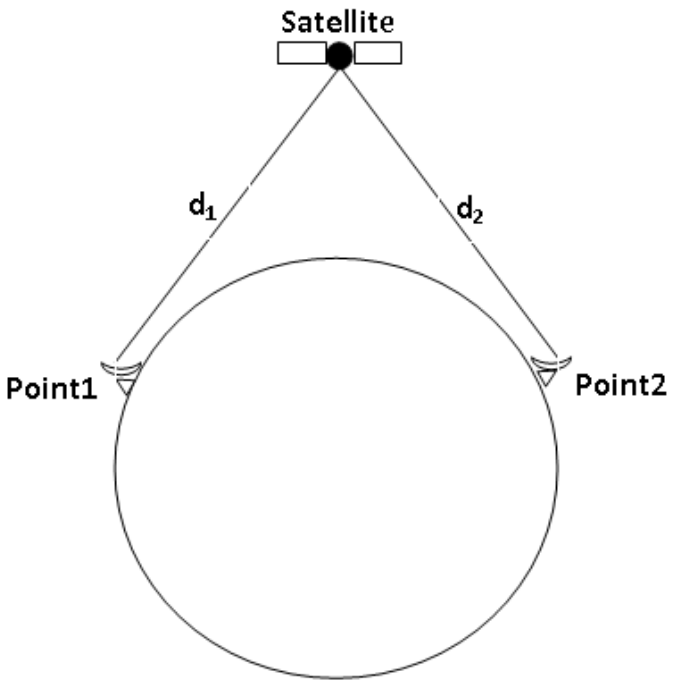

Figure 2: Distance between point 1 and point 2 on earth surface via geostationary satellite.

Let the distance between the two arbitrary points, say point 1 and point 2 on the earth surface, and the satellite be denoted by $d_{1}$ and $d_{2}$ respectively as shown Figure 2 . Then from equations (1), $d_{1}$ and $d_{2}$ can be expressed as given in eqns (3) and (4) respectively. The model equations for the propagation delays between each of these points and the satellite are expressed in eqns (5) and (6) respectively. Therefore, the model equation for the propagation delay between these two points via satellite is as expressed in eqn (7).

$$
\begin{aligned}
& d_{1}=\sqrt{D^{2}+R^{2}-2 D R \cos \left(\alpha_{1}\right) \cos \left(\Delta_{1}\right)} \\
& d_{2}=\sqrt{D^{2}+R^{2}-2 D R \cos \left(\alpha_{2}\right) \cos \left(\Delta_{2}\right)} \\
& \text { where: } \begin{aligned}
& \Delta_{1}=\Delta_{E S 1}-\Delta_{S} ; \\
& \Delta_{2}=\Delta_{E S 2}-\Delta_{S}
\end{aligned}
\end{aligned}
$$

$\Delta_{E S 1}=$ the angle of longitude of point 1 on the earth surface in degrees. 
$\Delta_{E S 2}=$ the angle of longitude of point 2 on the earth surface in degrees.

$$
\begin{aligned}
& T_{P 1}=\frac{d_{1}}{c} \\
& T_{P 2}=\frac{d_{2}}{c} \\
& T_{P 12}=T_{P 1}+T_{P 2}
\end{aligned}
$$

where $\alpha_{1}$ and $\alpha_{2}$ are the latitudes of point 1 and point 2 in degrees respectively. $\Delta_{E S 1}$ and $\Delta_{E S 2}$ are the longitudes of points 1 and 2 in degrees respectively. $\Delta_{1}$ and $\Delta_{2}$ are differences between subsatellite point longitude and longitudes of point 1 and point 2 in degrees respectively.

\section{MAPPING OUT THE BOUNDARY OF NIGCOMSAT-1R FOOTPRINTS}

Mapping out the geographical boundary of the footprints in terms of longitude and latitude becomes necessary in order to determine the range of propagation delay. The knowledge of the distance between the satellite and any point along the boundary of the satellite footprint is required for the determination of minimum and maximum possible propagation delay between the satellite and any points along the boundary of the satellite footprints. In a situation where the subsatellite point falls within the footprint location, its longitude and latitude will be part of the boundary data.

For the purpose of this work, the mapping was carried out empirically on NigComSat-1R footprints, which have been overlay with Google Map by Satbeams.com (SATBEAMS, 2017). The footprints boundary location longitude and latitude data were obtained by first logging into Satbeam home page through Google and then clicked on satellite footprint. The satellite footprint for all the geostationary satellite appeared and NigComSat-1R located at $42.5^{\circ}$ East was selected. The boundary of all the 9 footprints associated the satellite were mapped out one after the other by placing the cursor on the boundary location of interest and recording the displayed longitude and latitude.

The process was carried out by starting from a point on the boundary and moving stepwise along the boundary until one returned to the starting point. It should be noted that the closer the steps the more accurate the mapping. In other to confirm the conformal of the boundary generated using the data obtained with the actual boundary on the Google Map, trajectory of the boundary was plotted in MATLAB environment for each of the footprints using the values of longitude and latitude data generated along the boundary of each footprint region.

Figure 3(a) is the trajectory of boundary location for Cband ECOWAS 1 beam footprint traced using MATLAB while Figure 3(b) is the image of the actual boundary on the Google map. The generated boundaries for other footprint regions were also carried out in the same procedure. It can be seen from Figures 3(a) and 3(b), that there is a perfect matching between the generated boundary trajectories and the actual boundary of the footprints which is also the same for other footprint regions. This implies that the generated data are adequate for the determination of the distance between the satellite and any point along the boundary of the footprint regions. For easy of identification and reference, Table 1 represent the footprint regions and their respective label.

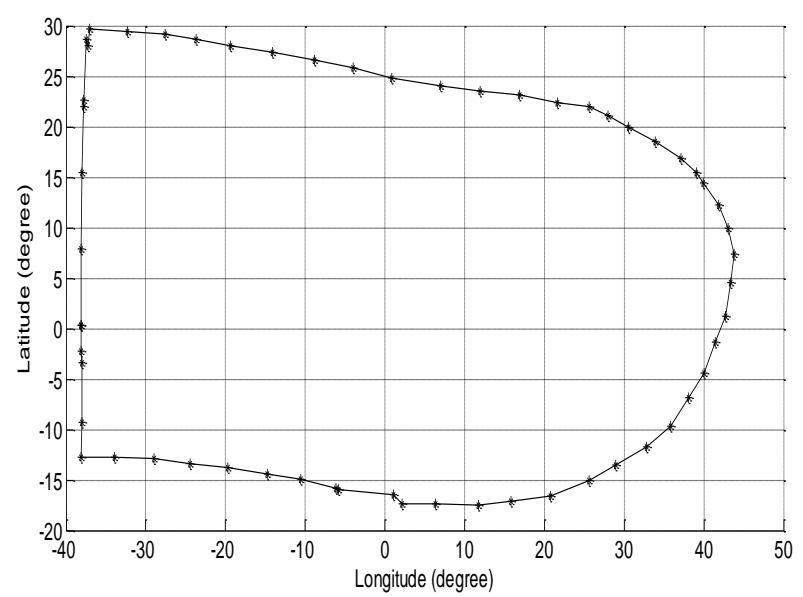

Figure 3a: Traced boundary location for C-band ECOWAS 1 beam footprint region.

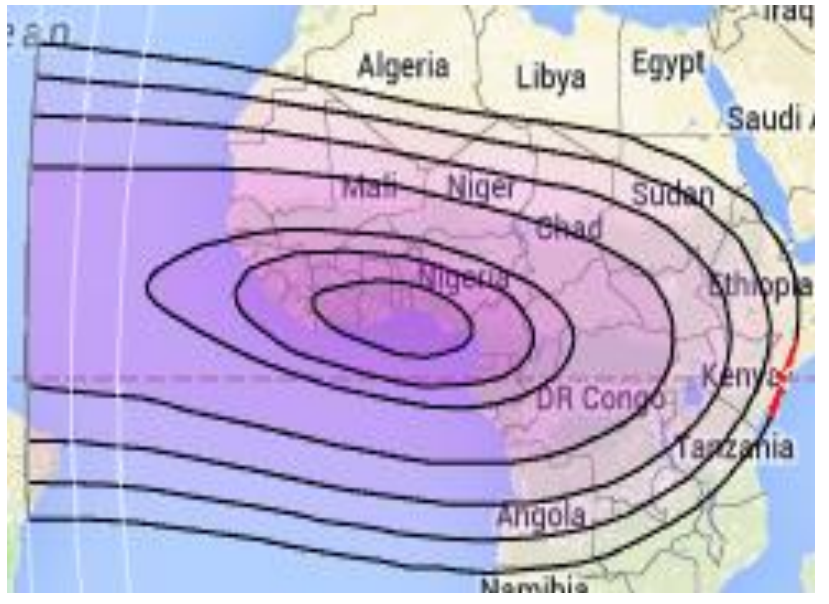

Figure 3b: Boundary location from Satbeams for C-band ECOWAS 1 beam footprint region.

Table 1: NigComSat-1R Footprint Regions.

\begin{tabular}{lc}
\hline Footprint Regions & Label \\
\hline C-band ECOWAS 1 beam & $\mathrm{A}$ \\
Ku-band ECOWAS 1 beam & $\mathrm{B}$ \\
Ku-band ECOWAS 2 beam & $\mathrm{C}$ \\
Ka-band Europe Spot beam & $\mathrm{D}$ \\
Ku-band Asian (KASHI) beam & $\mathrm{E}$ \\
L-band Navigation payload L1 beam & $\mathrm{F}$ \\
L-band Navigation payload L5 beam & $\mathrm{G}$ \\
Ka-band Nigeria Spot beam & $\mathrm{H}$ \\
Ka-band South Africa Spot beam & $\mathrm{I}$ \\
\hline
\end{tabular}




\section{SIMULATION OF PROPAGATION DELAY BETWEEN THE SATELLITE AND FOOTPRINTS BOUNDARY}

The propagation time delay between NigComSat-1R and the boundary of each of its footprint regions were simulated in MATLAB environment using equation (2); and the simulation graph for C-band ECOWAS 1 beam footprint region is as shown in Figure 4. Any point on the surface of this graph gives the location along the boundary of C-band ECOWAS 1 beam footprint region in terms of longitude and latitude and the associated propagation delay. From Figure 4, the minimum and maximum propagation delay were obtained as $0.1193 \mathrm{sec}$ and $0.1392 \mathrm{sec}$ respectively. Similar simulation graphs were plotted for the rest of the footprint regions and the resulting minimum and maximum propagation delays were also obtained from these graphs.

\section{RESULTS AND DISCUSSION}

From the simulation graph of Figure 4 and that of the remaining eight (8) footprint regions (not shown), the minimum and maximum possible propagation time delays for each of the footprint regions were determined and presented graphically as shown in Figures 5 and 6 respectively. As can be seen, the minimum and maximum possible time delay between the boundary of NigComSat-1R Footprints and the satellite are $0.1193 \mathrm{sec}$ and $0.1416 \mathrm{sec}$ respectively. The minimum possible delay was between C-band ECOWAS 1 beam footprint and the satellite while the maximum delay was between L-band Navigation payload L1 beam or L-band Navigation payload L5 beam and the satellite. This was because L1 beam and L5 beam have the same geographical footprint boundary region.

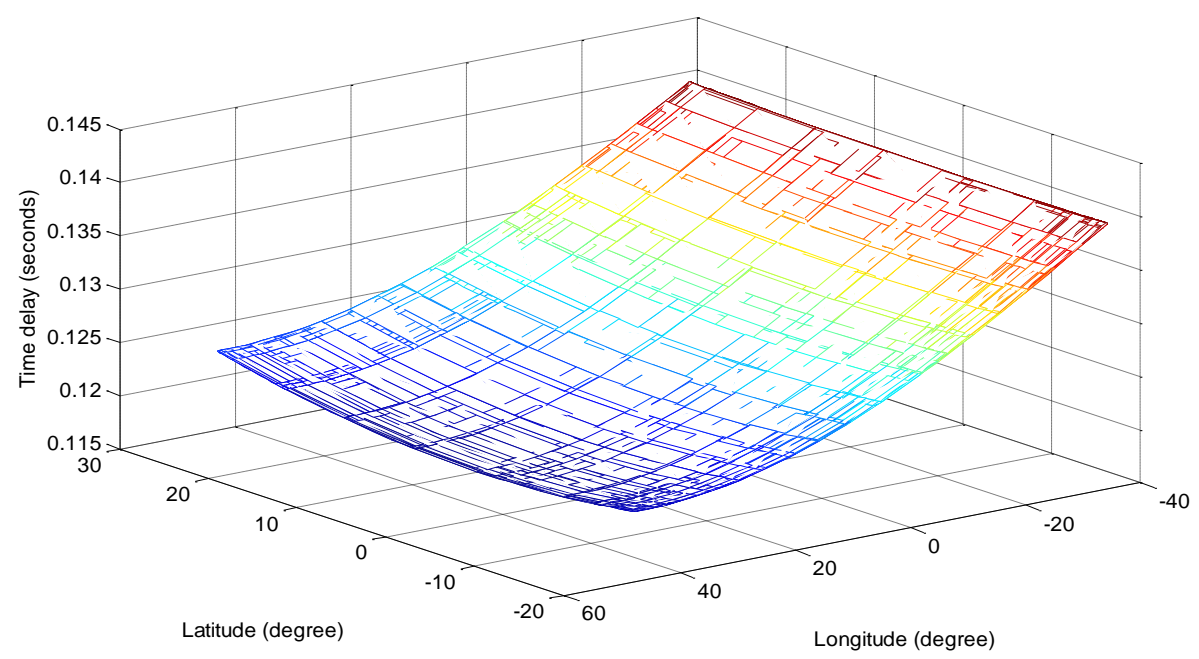

Figure 4: Simulation of propagation delay between satellite and boundary locations for C-band ECOWAS 1 beam footprint region.

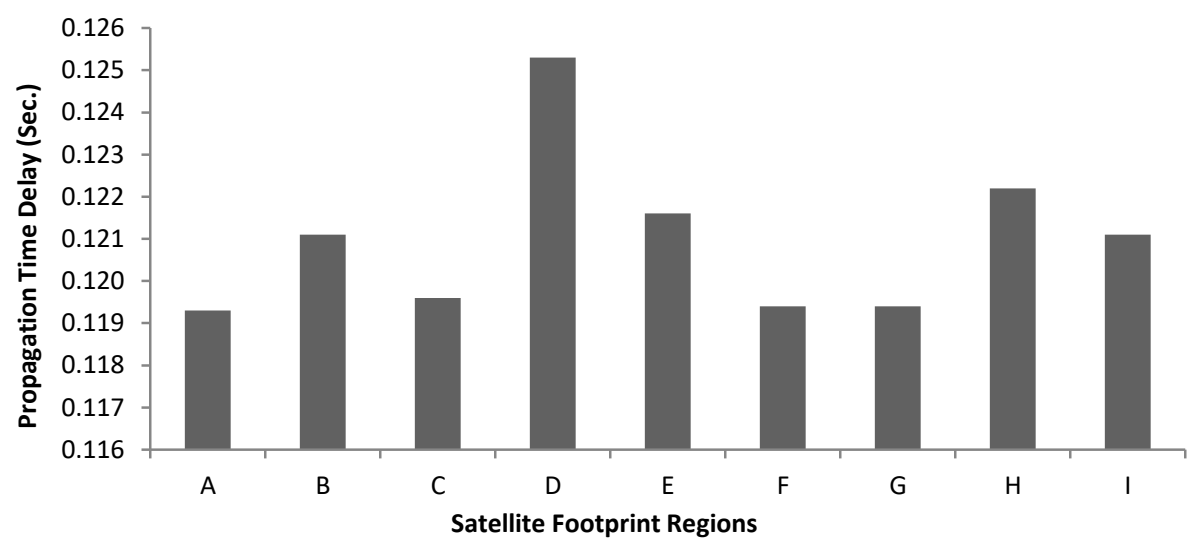

Figure 5: Minimum Propagation Delay between NigComSat-1R and its Footprints. 


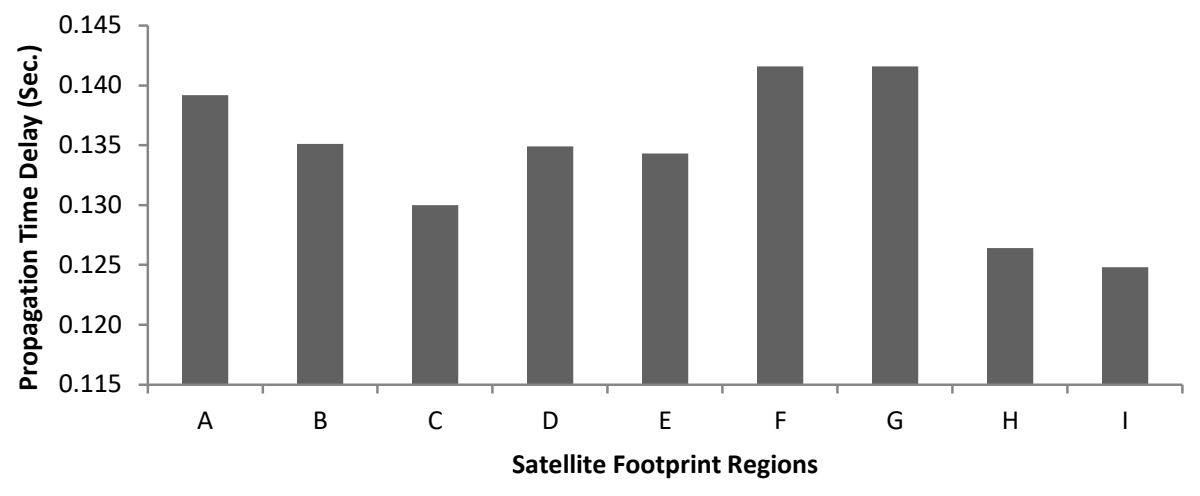

Figure 6: Maximum Propagation Delay between NigComSat-1R and its Footprints.

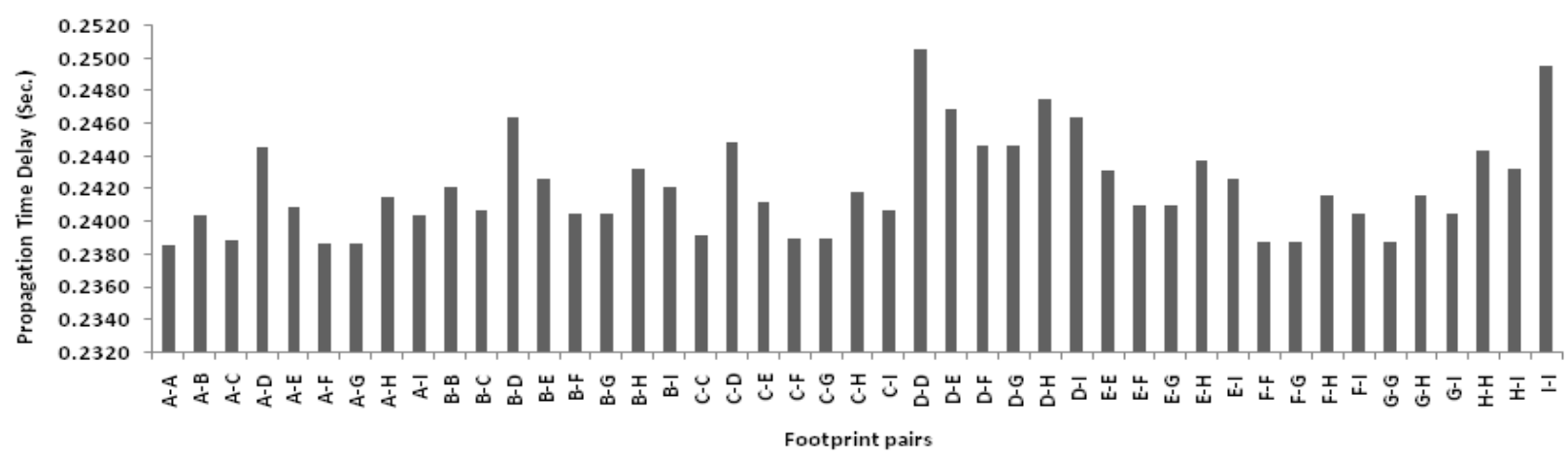

Figure 7: Minimum Propagation Delay between NigComSat-1R Footprint Regions via the Satellite

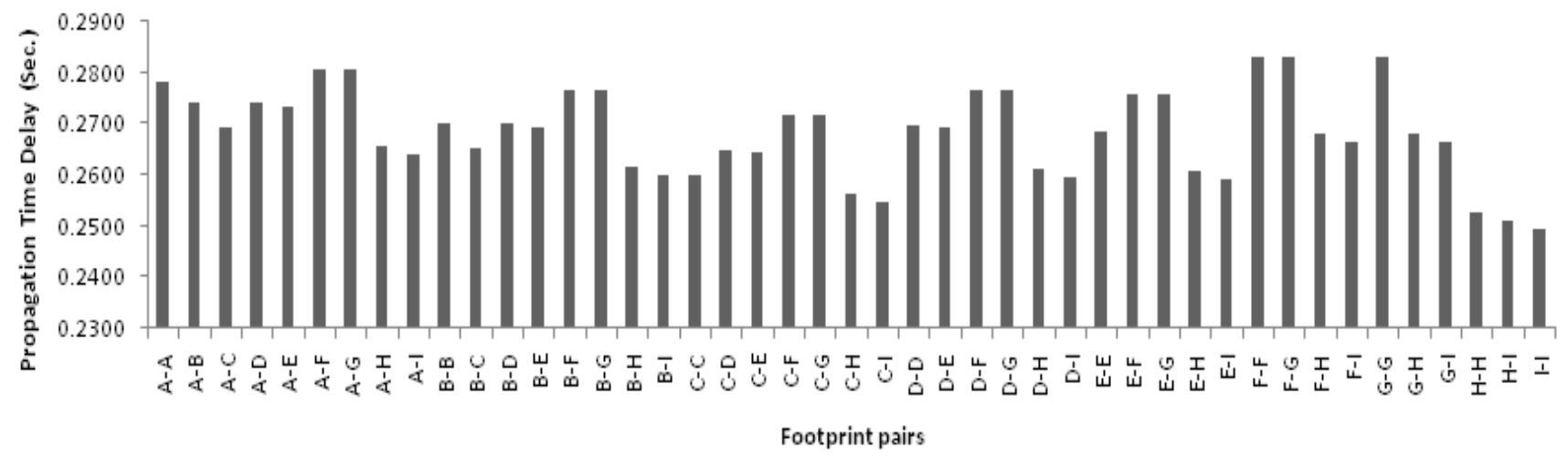

Figure 8: Maximum Propagation Delay between NigComSat-1R Footprint Regions via the Satellite.

To determine the minimum and maximum propagation time delays between any two Footprint regions of NigComSat-1R via the satellite, the minimum and maximum delay between each of the two footprint regions of interest and the satellite were obtained from Figures 5 and 6 respectively. The minimum and maximum propagation time delay between any two footprint regions of NigComSat-1R are then calculated by adding the corresponding minimum or maximum delays as shown in Figures 7 and 8 respectively. It was observed from Figure 7, that the minimum possible propagation time delay between any two footprint regions was between C-band ECOWAS 1 beam and itself and the value was $0.2386 \mathrm{sec}$, while from Figure 8 , the maximum possible propagation time delay between any two footprint regions was between L-band Navigation payload L1 beam and itself or L-band Navigation payload L5 beam and itself and the value was $0.2832 \mathrm{sec}$.

\section{CONCLUSION}

The model equation for determination of propagation time delays between any two footprint regions via geostationary satellite were derived. The minimum and maximum possible propagation time delays between NigComSat-1R and each of the footprint regions were determined. The results of minimum, maximum and range of propagation time delays are very crucial to the design and analysis of NCSs where NigComSat-1R serve as the link among control elements. The outcome of this research work 
will play a significant role in future research where the range of propagation delay associated with NigComSat-1R footprint regions may be required.

\section{REFERENCES}

Goyal, R.; R. Jain; M. Goyal; S. Fahmy; B. Vandalore and T. vonDeak (1999). Traffic Management in ATM Networks over Satellite Links. Technical Report, NASA Glenn Research Center; Cleveland, OH United States.

Ibiyemi, T. S., and Ajiboye, A. T. (2012a). Automatic Tracking of NigComSat-1R Satellite by Dish Network Mounted on Mobile Tele-Medicine Vehicles. International Journal of Engineering Research \& Technology, 1(4): 1-4.

Ibiyemi, T. S., \& Ajiboye, A. T. (2012b). On PID Controller Design for Mobile Node Network with Variable Delays. International Journal of Science and Advanced Technology, 2(4):176-180.

Khan, A.; D. Tilbury; and J. Moyne. (2008). Favorable effect of time delays on tracking performance of type-I control systems. IET Control Theory \& Applications, 2(3): 210-218.
Kolawole, M. O. (2002). Satellite communication engineering. Marcel Dekker New York.

Kumar, V., \& Kumar, R. (2013). Effect of NetworkInduced Delay on Stability in Networked Control System. International Journal of Scientific Research, 2(4):18-21.

Nwajiobi, E. N. (2012). The Nigerian Communications Satellite (NIGCOMSAT-1R): Relevance and Impact on Information and Communications Technology (ICT) Development in Nigeria and Africa . Journal of Research in Pure and Applied Sciences, 1(1): 28-36.

SATBEAMS. (2017). Footprints. Retrieved 16/04/2017,from http://www.satbeams.com/footprints

Vatanski, N.; J. P. Georges, C. Aubrun; E. Rondeau, and S-L. J. Jounela. (2006). Control compensation based on upper bound delay in networked control systems. arXiv preprint cs/0609151. 